\title{
Pediatric Transplantation in the United States, 1997-2006
}

\author{
J. C. Magee ${ }^{a, b}, *$, S. M. Krishnan ${ }^{c}$, M. R. \\ Benfield $^{d}$, D. T. Hsu ${ }^{e}$ and B. L. Shneider \\ a Department of Surgery, University of Michigan, Ann \\ Arbor, MI \\ b Scientific Registry of Transplant Recipients, Ann Arbor, \\ $\mathrm{MI}$ \\ 'Kidney Epidemiology and Cost Center/Scientific Registry \\ of Transplant Recipients, Ann Arbor, MI \\ 'University of Alabama Health System, Birmingham, AL \\ 'Montefiore Medical Center, Bronx, NY \\ $\mathrm{U}$ University of Pittsburgh, Pittsburgh, PA \\ * Corresponding author: John C. Magee, \\ mageej@umich.edu
}

This article represents the sixth annual review of the current state of pediatric transplantation in the United States from the Scientific Registry of Transplant Recipients (SRTR). It presents updated trends, discussion of analyses presented during the year by the SRTR to the committees of the Organ Procurement and Transplantation Network (OPTN) and discussion of important issues currently facing pediatric organ transplantation. Unless otherwise stated, the statistics in this article are drawn from the reference tables of the 2007 OPTN/SRTR Annual Report. In this article, pediatric patients are defined as candidates, recipients or donors aged 17 years or less. Data for both graft and patient survival are reported as unadjusted survival, unless otherwise stated (adjusted patient and graft survival are available in the reference tables). Short-term survival $(3 \mathrm{month}$ and 1 year) reflects outcomes for transplants performed in 2004 and 2005; 3-year survival reflects transplants from 2002 to 2005; and 5-year survival reports on transplants performed from 2000 to 2005 . Details on the methods of analysis employed may be found in the reference tables themselves or in the technical notes of the 2007 OTPN/SRTR Annual Report, both available online at wWw.ustransplant.org.

Key words: Adherence, heart transplantation, kidney transplantation, liver transplantation, lung transplantation, pediatric transplantation

\section{Pediatric Organ Donors}

Organ donation in the pediatric population has declined somewhat in the past 10 years, both in absolute numbers (from 1056 in 1997 to 966 in 2006) and in percentage of total donors (from 19.3\% in 1997 to $12.0 \%$ in 2006). The distribution of pediatric donor ages has remained consistent, with close to $60 \%$ between the ages of 11 and 17 . Among deceased donors of kidneys, the number and percentage of pediatric donors declined between 1997 and 2006 (from 967 to 886 and from $19.0 \%$ to $12.3 \%$, respectively). The age distribution of pediatric kidney donors has remained consistent. The number of pediatric pancreas donors grew from 305 in 1996 to 388 in 2006, while the percentage of pediatric donors declined from $23.1 \%$ to $19.2 \%$. The age distribution has fluctuated somewhat, but the largest group of pediatric pancreas donors has been among ages 11-17 (81-89\% each year). While the total number of liver donors has increased over the past 10 years, the number of pediatric liver donors has decreased from 939 to 860 (from $20.4 \%$ to $12.3 \%$ ). As with other organs, donors age 11-17 make up the largest fraction of pediatric liver donors. The number of pediatric intestine donors has increased from 58 to 128 , but no consistent trend in percentage has been observed. The number of pediatric heart donors has declined from 585 to 474 (from $24.1 \%$ to $20.8 \%$ ), whereas the number of adult heart donors has remained steady. There is no apparent trend in the absolute number of pediatric lung donors, but the percentage of pediatric lung donors has declined from $22.7 \%$ to $15.8 \%$.

While the number of adult donors is significantly greater than the number of pediatric donors, the percentage of organs transplanted versus organs recovered from deceased donors is generally greater within the pediatric age range, even when the analysis is restricted to young and middle age adults (donors aged 18 to 49 years). For deceased kidney donors, $94.7 \%$ of the pediatric kidneys recovered were transplanted while $91.6 \%$ of the kidneys recovered from donors age 18 to 49 years were transplanted. There was a similar trend for pancreas donors $(81.4 \%$ vs. $72.2 \%)$, liver donors (96.0\% vs. 91.4\%) and intestine donors $(94.7 \%$ vs. $85.7 \%$ ). With heart and lung donors, the percentage of organs transplanted that were recovered is high and similar for both pediatric donors and adult donors aged 18 to 49 years $(98.9 \%$ vs. $99.0 \%$ for heart and $98.1 \%$ vs. $96.4 \%$ for lung) (SRTR Analysis December 2007).

The ever present shortage of deceased donors has led to a broad range of efforts to increase donation, including donation after cardiac death (DCD). Over the last decade, DCD has increased across all age ranges; from 78 in 1997 to 


\section{Magee et al.}

647 in 2006 (1.4\% to $8.1 \%$, respectively). In the pediatric population, the number of DCD donors increased from 6 to 77 . Less than $1 \%$ of the pediatric donors were DCD in 1997; in 2006, approximately $8 \%$ of pediatric donors were DCD. While the relative percentage of DCD donors is similar in both the adult and pediatric population, there is likely an unmet potential of DCD in the pediatric population (1). Recent policies advanced by both The Joint Commission (JCAHO) and the Organ Procurement and Transplantation Network (OPTN) requiring hospitals to develop and implement DCD policies have refocused interest on DCD. In moving forward with policy development, many institutions have faced challenges related to the fact that DCD is relatively unfamiliar in pediatric ICUs, where welldeveloped end-of-life and withdrawal of support practices already exist. Additionally, the relatively small numbers of pediatric DCD donors and transplant recipients within any hospital make informed discussion of the challenges and solutions more problematic.

It is important for the pediatric transplant community to actively engage those involved in end-of-life care for children and to provide perspective and guidance. Developing and sharing best practice models for pediatric DCD may help. As a step forward, the Health Resources and Services Administration (HRSA), the Organ Donation and Transplantation Alliance and the United Network for Organ Sharing (UNOS) sponsored a Pediatric Summit on Organ Donation and Transplantation in San Antonio, Texas in March 2007. This summit brought together members of the pediatric ICU and transplant communities to discuss solutions to facilitate donation in the pediatric population. This effort will hopefully enhance inter-disciplinary dialogue and assist institutions in developing pediatric DCD programs. While efforts to increase DCD are worthwhile because they will result in more donors, it is also important to realize that DCD provides the family of a child contemplating withdrawing life support because of irreversible neurologic injury the opportunity to pursue organ donation, if that is their wish.

\section{Overall Pediatric Waiting List}

The total number of pediatric candidates on the waiting list at a given point in time increased steadily from 1739 in 1997 to a peak of 2340 in 2001 and has since leveled, with 2124 candidates in 2006 (Figure 1). Distribution by age of pediatric candidates on the waiting list has remained consistent, averaging $6.6 \%$ aged less than 1 year, 26.9\% 1-5, 19.8\% 6-10 and 46.7\% 11-17 years. The overall percentage of pediatric candidates on the waiting list has declined somewhat, from 3.3\% in 1997 to $2.3 \%$ in 2006.

The number of pediatric candidates active on the liver waiting list increased steadily in the late 1990s to a peak of 703 in 2001. It then dropped to 537 in 2002 and has since declined steadily to 361 in 2006 . This trend was similar to

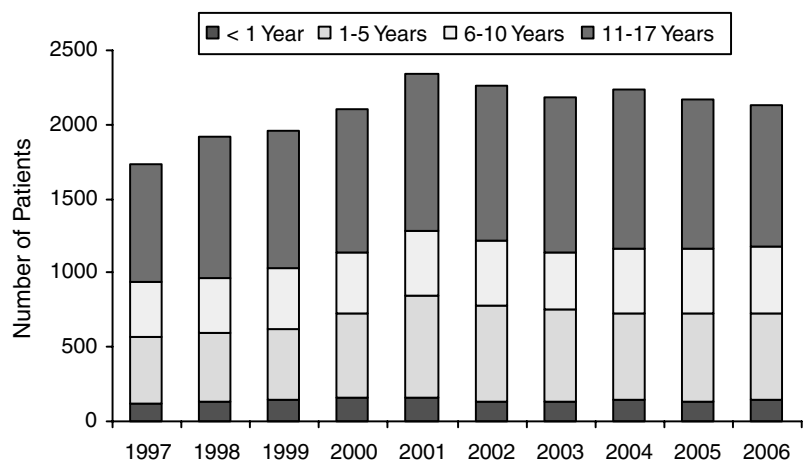

Source: 2007 OPTN/SRTR Annual Report, Table 1.4.

Figure 1: Pediatric transplant waiting list (active and inactive) at year-end for all organs, by age, 1997-2006.

that of the number of adults on the liver waiting list. Data regarding the kidney waiting list were similar in both trend and number: after a steady increase, the number of candidates peaked at 645 in 2002, then dropped to 559 in 2003 and dropped further to 422 in 2006.

The number on the intestine waiting list almost tripled over the decade, from 53 in 1996 to 140 in 2006 . There has been no obvious trend in the number of active pediatric candidates on the heart waiting list, with about 85-110 waiting at the end of each year. The total number of candidates of any age on the heart waiting list decreased from 2414 in 1997 to 1327 in 2006. The active pediatric candidates on the heart waiting list made up only $3.5 \%$ of the waiting list in 1997; this percentage had increased to about $8 \%$ in 2006. The number of pediatric candidates on the lung waiting list was nearly constant (approximately 130 each year) between 1997 and 2004, but dropped to 77 in 2005, which further dropped to 42 in 2006 . The changes in the pediatric waiting list stratified by organ type are illustrated in Figure 2.

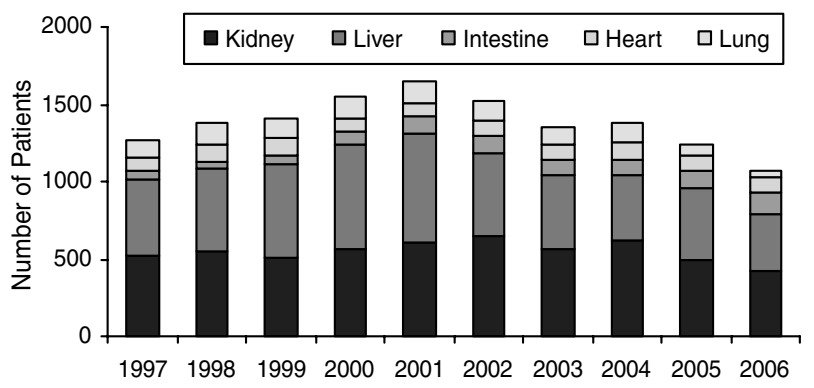

Source: 2007 OPTN/SRTR Annual Report, Tables 5.1a, 9.1a, 10.1a, 11.1a, and 12.1a.

Figure 2: Distribution of active pediatric waiting list candidates at year-end, by organ, 1997-2006. 


\section{Pediatric Transplant Recipients}

Over the last 10 years, the number of pediatric recipients of any organ grew 20\% (from 1648 to 1979), in contrast to the $44 \%$ increase in the number of adult recipients (from 18657 to 26951 ). The greatest increase, $35 \%$, occurred in the largest group, recipients 11-17 years old (648 in 1997 to 876 in 2006). The remaining pediatric groups experienced increases of smaller proportion: recipients $<1$ year old increased $13 \%$ (278 to 313 ) and recipients $1-5$ years old increased $16 \%$ (447 to 517 ). The number of transplants in the 6- to 10-year olds decreased by 1\% from 275 in 1997 to 273 in 2006 . As on the waiting list, the age distribution among transplant recipients has remained consistent at about $16 \%$ under 1 year, 26\% $1-5$ years, 16\% 6-10 years and $42 \% 11-17$ years. The proportion of pediatric patients among recipients has declined by approximately $15 \%$ from $8.1 \%$ in 1997 to $6.8 \%$ in 2006 .

\section{Kidney Transplantation}

Kidney transplantation is well accepted as the optimal therapy for children with end-stage renal disease (ESRD). In efforts to prioritize allocation to children awaiting kidney transplantation, over much of the last decade the generally agreed-upon underlying principle regarding deceased donor allocation has been to try to transplant children from 0 to 6 years old within 6 months, children from 7 to 12 years within 12 months and patients 12 to 18 years within 18 months. While this resulted in shorter waiting times for children compared to adults, it still required a waiting period. As these children still received relative priority, this approach conferred little benefit to anyone awaiting transplantation. Additionally, once these time goals were reached, it was common practice for centers to wait for better donor offers, further compounding the waiting time.

A Scientific Registry of Transplant Recipients (SRTR) analysis, performed in conjunction with the OPTN KidneyPancreas Committee, demonstrated that the characteristic most associated with improved outcomes in deceased donor pediatric transplantation was donor age between 5 and 35 years (2). Consequently, in 2005 the OPTN implemented a pediatric kidney allocation policy under which relative priority for kidneys from deceased donors less than 35 years old was assigned to recipients less than 18 years old, after any zero mismatch transplants, recipients with a PRA $>80$, or candidates receiving a kidney with a nonrenal organ (3).

One anticipated effect of this policy is a reduction in deceased donor waiting time for pediatric recipients. While pediatric candidates have always had significantly shorter waiting times compared to adults, there has been a further reduction in median waiting times observed following implementation of this policy (Figure 3). This effect is most pronounced in the 11- to 17-year-old group, likely reflect-

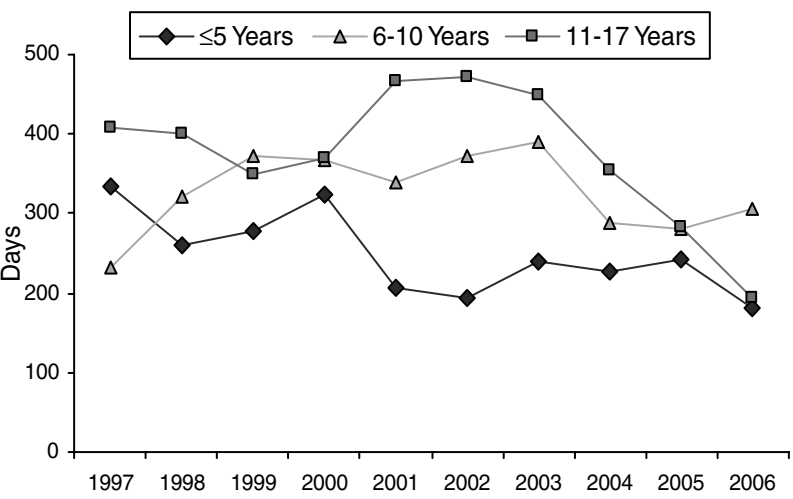

Source: 2007 OPTN/SRTR Annual Report, Table 5.2.

Figure 3: Median time to transplant for new kidney transplant waiting list registrants, by age, 1997-2006.

ing the longer waiting times in these older children under the previous time-based targets. Because these times to transplant are based on year of listing, the new policy has affected recipients listed in the years prior to its implementation. It is unknown where the new median time to transplant will reequilibrate.

While there has been a reduction in waiting time, there has been little change in the age distribution of pediatric candidates on the kidney waiting list. Additionally, children on the kidney waiting list have consistently had the lowest death rates with a rate of about 10 deaths per 1000 patientyears in 2006.

While the new allocation policy appears to have significantly increased access for deceased donor kidneys, it may also be contributing to a change in practice in living donor and deceased donor transplantation (Figure 4). Over most of the last decade, living donor transplants accounted for slightly more kidney transplants to pediatric recipients than deceased donor, non-expanded criteria donor (ECD) transplants. This changed in 2005, when there were 422 living donor transplants and 468 deceased donor, non-ECD transplants. This difference further widened in 2006, when there were 312 living donor compared to 581 deceased donor, non-ECD transplants. Pediatric kidney recipients are rarely transplanted with ECD kidneys. There was only one such pediatric recipient in 2006.

In the past decade, there has been a 2-fold increase in the number of deceased donor non-ECD transplants to pediatric recipients. In 2006 alone, the number of these transplants to pediatric recipients increased $24 \%$ to 581 , from 468 in 2005 . There is great concern that this most recent increase in deceased donor transplantation is a result of the higher priority granted for deceased donors, which has resulted in a reduction of living donor transplantation. Although there were increases in the number of deceased donor transplants to children $6-10$ and $<5$ years old, most 


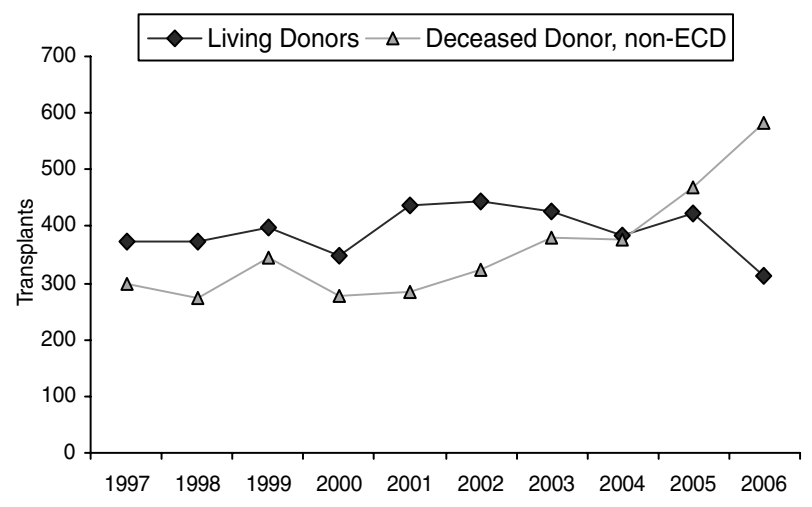

Source: 2007 OPTN/SRTR Annual Report, Table 5.2.

Figure 4: Pediatric kidney transplant recipients by donor type, 1997-2006.

of the absolute increase is attributable to a $25 \%$ increase in deceased donor transplants to the 11-17 group (Figure 5). Additionally, it appears that the recent shift from living donors to deceased donors is primarily a phenomenon affecting these older children. It is likely that at least some of this apparent shift reflects a 'bolus effect' of deceased donor transplants in these older children, and is reflective of higher allocation priority rather than an exclusive change in practice patterns to not pursue living donation. However, no doubt there have also been instances where a child was listed while living donors were being evaluated and a deceased donor became available. Whether this trend will continue remains to be determined. Given the increased availability of deceased donor kidneys, pediatric centers may need to alter listing practices while potential living donors are being evaluated. One would hope that given the better results following living donor transplant, centers would exhaust this option prior to accepting a deceased donor kidney.

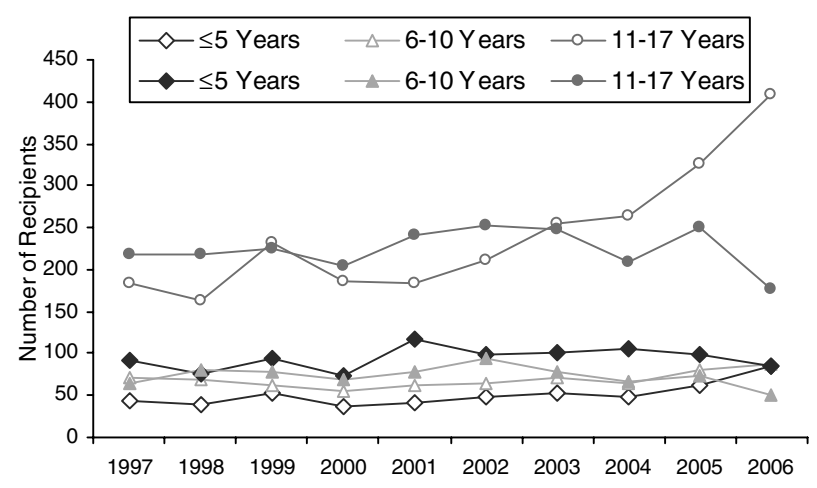

Closed symbol = living donor; open symbol = deceased donor, non-ECD. Source: 2007 OPTN/SRTR Annual Report, Table 5.4a and 5.4c.

Figure 5: Pediatric transplant recipients, by donor type, by age, 1997-2006.

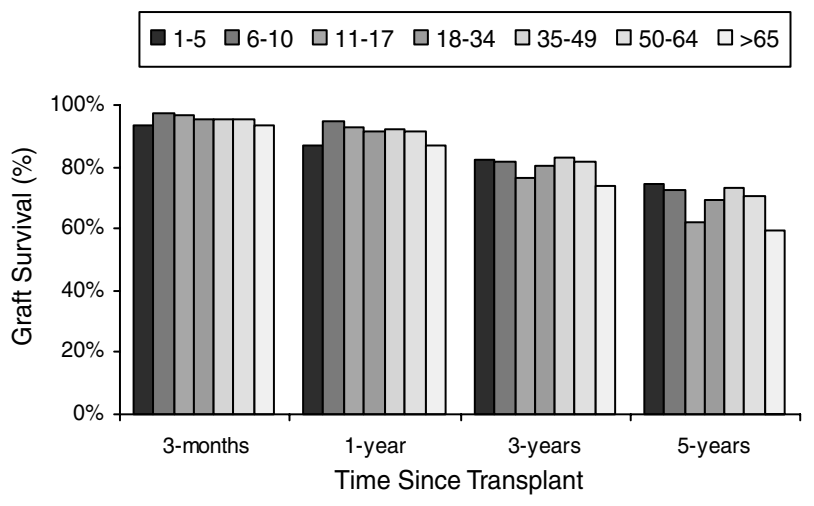

Source: 2007 OPTN/SRTR Annual Report, Table 5.10a.

Figure 6: Unadjusted graft survival of deceased donor nonECD kidney transplants, by recipient age.

Outcomes following pediatric kidney transplantation are highly dependent on donor source (Figure 6 and Figure 7). Overall, living donor pediatric kidney recipients had better unadjusted 1-, 3- and 5-year graft survival than the deceased donor, non-ECD recipients. Three-year graft survival was markedly higher for living donor recipients compared to deceased donor, non-ECD recipients for pediatric recipients $1-5$ years old (93\% vs. $82 \%$ ), 6-10 years old ( $92 \%$ vs. $82 \%$ ) and $11-17$ years old ( $85 \%$ vs. $76 \%$ ). The graft survival advantages associated with living donor recipients was even greater in 5-year graft survival across pediatric recipients $1-5$ years old ( $89 \%$ vs. 75\%), 6-10 years old $(85 \%$ vs. $72 \%)$ and $11-17$ years old (74\% vs. $63 \%)$. Consistent with previous reports, while short-term results are excellent in the 11- to 17-year-olds, there is a significant increase in graft loss compared to other recipients several years posttransplant (4).

1-5 $\square$ 6-10 $\square$ 11-17 $\square$ 18-34 $\square$ 35-49 $\square$ 50-64 $\square>65$

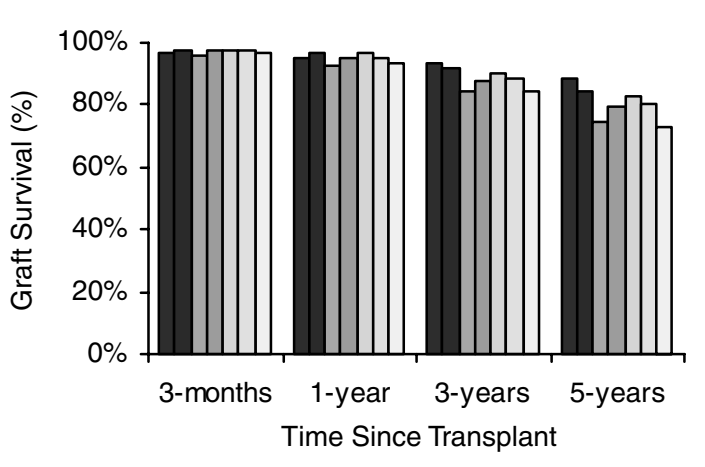

Source: 2007 OPTN/SRTR Annual Report, Table 5.10c.

Figure 7: Unadjusted graft survival of living donor kidney transplants, by recipient age. 
While some worry that the recent policy changes providing greater access to deceased donors will lower the likelihood of a highly matched kidney, Gritsch et al. independently reported that, except for zero mismatched allografts, the impact of HLA matching in deceased donor pediatric kidney transplantation was minor (5). While it is likely this finding will remain consistent for recipients of deceased donor kidneys following the policy change, the follow-up time is insufficient to conduct a meaningful analysis of long-term outcomes. Nonetheless, it is likely that given the better outcomes following living donor transplants compared to deceased donors, any practice associated with an increase in deceased donor transplants may actually decrease overall graft survival across the population.

With respect to patient survival, there were no striking differences for living donor recipients in the different pediatric age groups, with 5-year survival in the range of 94.796.3\%. Patient survival rates in living donor kidney recipients were slightly better for the 6- to 10-year-olds and 11 to 17-year-olds compared to the 1- to 5-year-olds. Unadjusted patient survival for deceased donor, non-ECD recipients was slightly lower than that of living donor recipients. While recipient survival is excellent, there remains a real risk of mortality, which has not changed significantly over recent years. The number of patients living with a functioning kidney transplant who received their transplants as children increased over the decade to 5338 recipients in 2005.

\section{Liver Transplantation}

The number of patients of all ages who are active on the liver waiting list increased from 7571 in 1997 to a peak of 14893 in 2001 and has leveled off at about 12500 since then. The number of pediatric patients on the waiting list grew steadily from 492 in 1997 to a peak of 703 in 2001 and has since declined to 361 in 2006 . The pediatric patients on the waiting list now make up a much smaller proportion of the list (2.9\% compared to $6.5 \%$ in 1997$)$ because of the overall growth on the list.

Pediatric liver candidates account for a greater percentage of all candidates who are inactive on the liver waiting list $(8.9 \%)$ compared to those active $(2.9 \%)$ on the liver waiting list in 2006. At the end of 2006, 48.6\% of pediatric candidates were active, compared to $75.6 \%$ of adult candidates. The reasons why there are significant numbers of pediatric candidates inactive at any given time compared to adult candidates is unknown. The numbers and percentage of both pediatric and adult liver transplant candidates that were inactive at the end of 2006 is summarized by OPTN region in Table 1 . There is evidence of significant regional variation, which may reflect centerspecific practices. These findings are worthy of further investigation.
Table 1: Inactive pediatric and adult liver transplant candidates as of 31 December 2006, by OPTN region

\begin{tabular}{|c|c|c|c|c|}
\hline & \multicolumn{2}{|c|}{ Pediatric } & \multicolumn{2}{|c|}{ Adult } \\
\hline & $n$ & $\%$ & $n$ & $\%$ \\
\hline Overall & 382 & 51.4 & 3931 & 24.4 \\
\hline Region 1 & 24 & 80.0 & 438 & 42.9 \\
\hline Region 2 & 24 & 13.3 & 542 & 24.3 \\
\hline Region 3 & 11 & 25.0 & 178 & 19.3 \\
\hline Region 4 & 20 & 55.6 & 290 & 19.6 \\
\hline Region 5 & 174 & 77.3 & 790 & 20.3 \\
\hline Region 6 & 6 & 42.9 & 74 & 19.2 \\
\hline Region 7 & 42 & 66.7 & 295 & 19.4 \\
\hline Region 8 & 21 & 53.8 & 211 & 23.9 \\
\hline Region 9 & 38 & 69.1 & 536 & 28.9 \\
\hline Region 10 & 9 & 27.3 & 178 & 23.2 \\
\hline Region 11 & 13 & 56.5 & 399 & 34.2 \\
\hline
\end{tabular}

The death rate for pediatric candidates on the liver waiting list is highly dependent on age (Figure 8). While children above 6 years of age have equivalent or slightly lower death rates compared to adults, younger children have the highest death rates of all candidates. Additionally, it appears that for the last 2 years, the death rate while awaiting transplantation may be increasing in children less than 6 years of age. While the small numbers of events make analysis difficult, there were 103 pediatric candidates who died in 2006 prior to transplantation.

Recent allocation changes in liver transplantation most relevant to the pediatric population include the use of model for end-stage liver disease (MELD) rather than pediatric end-stage liver disease (PELD) for allocation in adolescent candidates, and the refinement of the criteria for Status 1 listing, with the creation of Status $1 \mathrm{~A}$ and $1 \mathrm{~B}$ for pediatric candidates. These Status 1 designations were accompanied by more rigorously defined criteria for who qualifies for listing under each status. Status $1 \mathrm{~B}$ is reserved for pediatric candidates with chronic liver disease who are in the ICU with a calculated PELD score of $>25$ or calculated MELD

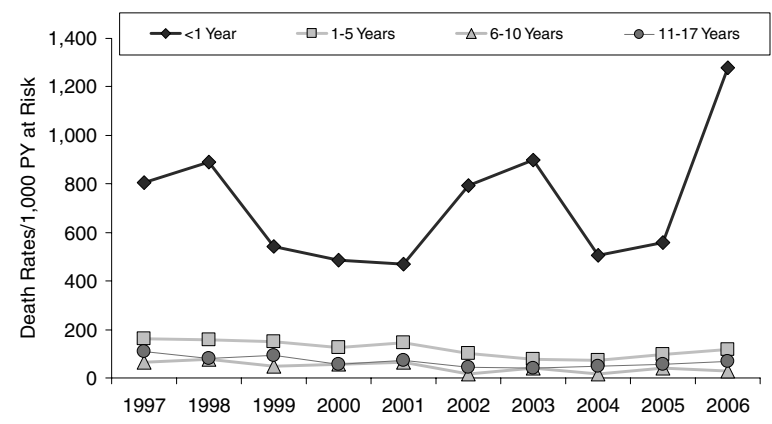

Source: SRTR Analysis, June 2007

Figure 8: Reported pediatric liver only (no intestine) waiting list annual death rates per 1000 patient-years at risk, 19972006. 
score of $>25$ for adolescent candidates (12-17 years) and are also on a ventilator, mechanical ventilator, have had gastrointestinal bleeding requiring at least $30 \mathrm{cc} / \mathrm{kg}$ of red blood cell replacement within the previous 24 hours, have renal failure requiring dialysis, or have a Glasgow coma scale < 10. One factor in the decision to allow children with chronic liver disease to be listed as a Status 1B was based on an SRTR analysis that showed that when these children had a PELD score $>25$ and were listed as a Status 1 by exception, their observed mortality rate was significantly higher than reflected by their PELD score alone.

While the use of MELD for adolescent candidates was shown in an SRTR analysis to usually result in a higher allocation score than they would receive using PELD, demonstrating any significant impact on outcomes due to this change will be difficult given the relatively limited size of this group. Similarly, the small number of pediatric patients listed as Status 1B to date limits the ability to demonstrate any significant impact on outcomes. In 2006, the first full year after the policy change, there were 46 liver transplants performed for Status $1 \mathrm{~B}$ recipients. After several years of experience, a more in-depth analysis may be possible.

As noted in the intestine section, there are efforts to further refine liver allocation and preferentially allocate from the smallest deceased donors to candidates listed for liverintestine transplantation over liver alone due to a high risk of waiting list mortality in the liver-intestine population that is not well reflected by their PELD score. While it is hoped that such efforts will decrease mortality in the small children awaiting liver-intestine transplantation, it is possible that this preferential allocation will adversely affect the waiting list mortality rate in the youngest candidates awaiting liver transplantation, a sub-population that also faces a disproportionately high mortality rate. While candidates awaiting liver transplantation alone have the potential to receive technical variant grafts from either deceased or living donors, it is unknown if this option will be sufficient. It is clear, based on a previous analysis, that the use of deceased donor technical variant grafts is associated with a higher risk of graft failure and mortality compared to whole organ grafts $(6,7)$.

The number of deceased and living donor liver transplants to pediatric candidates has been reasonably constant, with 511 deceased donor and 64 living donor transplants in 2006 (Figure 9). The introduction of adult-to-adult living donor liver transplantation has significantly impacted who receives a living donor liver transplant. In 1998, the percentage of all living donor liver transplants that went to adults was about $27 \%$; in 2006, the reverse was true, and $22 \%$ of living donor liver transplants went to pediatric candidates.

Unadjusted graft survival at 1 year for pediatric living donor liver recipients under 6 years of age was better than deceased donor liver recipients (for < 1-year-olds: $88 \%$ vs. $86 \%$; for $1-5$ : $91 \%$ vs. $81 \%$ ). Among the same population,

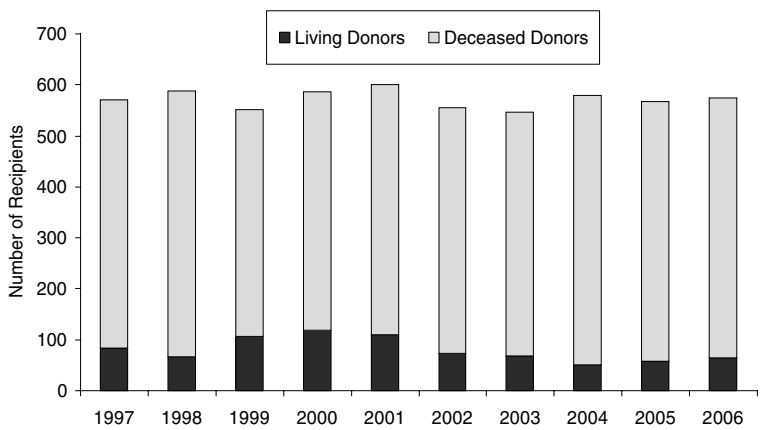

Source: 2007 OPTN/SRTR Annual Report, Tables 9.4a and 9.4b.

Figure 9: Pediatric recipients of deceased and living donor liver transplants, 1997-2006.

graft survival at 3 years was also slightly better for those who received living donor livers than for recipients of deceased donor livers (for < 1: $83 \%$ vs. $80 \%$; for $1-5$ : $79 \%$ vs. $76 \%)$.

The last decade has seen significant progress in pediatric liver transplantation, as evident by evaluation of the death rates in the first year following transplantation (Figure 10). While there has been improvement in survival across all ages, the improvement is most striking in the children less than 1 year of age. As another example of progress, there have been no deaths in the first year posttransplant in the 83 recipients with a diagnosis of biliary atresia who underwent living donor transplantation over the last 3 years.

Among deceased donor pediatric recipients, patient survival at 5 years was highest for the 6- to 10-year-old group at $89 \%$. Given that the number of liver transplants to adults has increased while the number to pediatric candidates has remained steady, it is not surprising that, though the number of pediatric (at the time of transplant) recipients alive with a functioning liver increased 62\% from 2835 in 1997 to 4605 in 2005, their

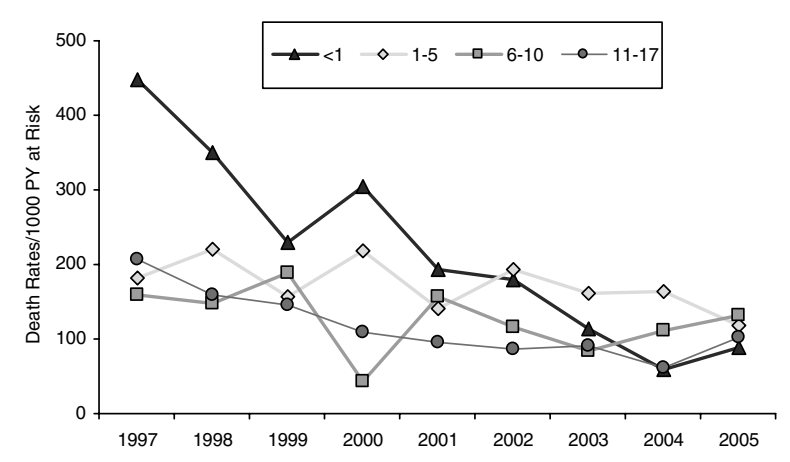

Source: 2007 OPTN/SRTR Annual Report, Table 9.7a.

Figure 10: Death rates for pediatric recipients during first year after deceased donor liver transplantation, 1997-2005. 
proportion of the overall population with functioning liver transplants has decreased from $15.1 \%$ to $12.6 \%$.

\section{Intestine Transplantation}

The number of pediatric candidates on the intestine waiting list almost tripled over the decade, from 53 in 1997 to 140 in 2006, and children remained at a consistent proportion, making up about $77 \%$ of the overall list. In 2006, $42.8 \%$ of pediatric intestine candidates were listed for intestine alone compared to $61.9 \%$ for adult candidates. The higher rate of listing for liver-intestine transplantation in the pediatric age range may reflect differences in the primary diagnosis leading to the need for intestine transplantation. Annual death rates on the intestine waiting list have varied extensively, and the rate is highly dependent on the relative number of candidates awaiting liver-intestine transplantation. For candidates listed initially for intestine alone, across all age ranges the death rate in 2006 was 379 per 1000 patient-years. The number of children dying while awaiting intestine transplantation has remained constant. The number of intestine transplants to pediatric recipients has increased about 200\% from 46 in 1997 to 95 in 2006. This trend is seen in all the pediatric age groups, the most significant increase being in the number of $<1$-year-olds receiving intestine transplants. Five-year graft survival is poor for intestine recipients, at 39\% for < 1-year-olds, 54\% for 1 - to 5 -year-olds, $45 \%$ for 6 - to 10 -year-olds and $48 \%$ for 11 - to 17 -year-olds. Patient survival at 5 years was $48 \%$ for $<1$-year-olds, $58 \%$ for 1 - to 5 -year-olds, $70 \%$ for 6 - to 10 year-olds and $67 \%$ for 11 - to 17 -year-olds (Figure 11). Graft and patient survival rates for pediatric intestine recipients at 1,3 and 5 years improved with increasing age. Among the pediatric intestine transplant recipients, 11- to 17-yearolds had the best graft and patient survival rates. At the end of 2005, there were 311 pediatric intestine recipients living with functioning transplants.

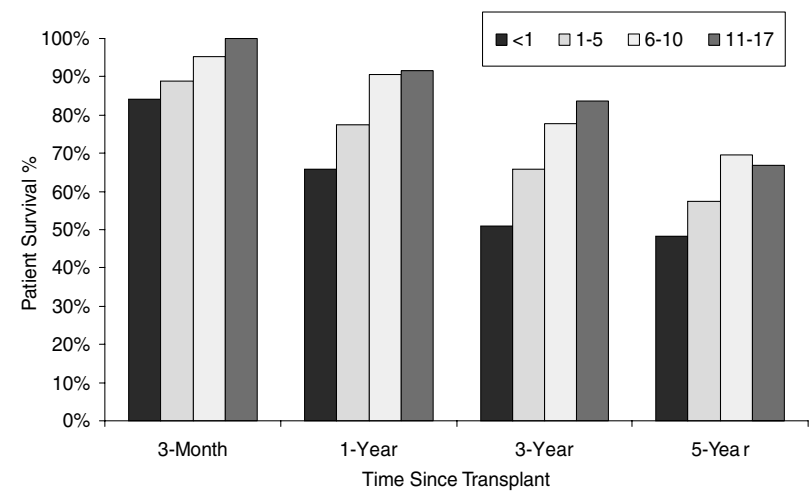

Source: 2007 OPTN/SRTR Annual Report, Table 10.14.

Figure 11: Unadjusted patient survival at 3 months, 1 year, 3 years and 5 years following intestine transplant, by age.
Previous reports have discussed in detail the high death rate for candidates awaiting intestine transplantation and how these candidates seem less well served by PELD or MELD compared to liver alone candidates $(4,8)$. Recent allocation changes have included giving pediatric candidates listed for liver-intestine transplantation a 23-point increase in their PELD or MELD score. Other considerations are policies that may direct small donor livers (if suitable for use as a composite allograft with intestine) to the infants listed for both organs rather than to children requiring a liver transplant alone.

\section{Heart Transplantation}

Although the number of candidates on the heart waiting list has decreased from 2414 in 1997 to 1327 in 2006, the number of pediatric candidates has been nearly constant, with 106 active on the waiting list at the end of 2006. The number of new pediatric heart registrants by age category has also remained relatively constant (Figure 12). While the number of new pediatric registrants on the heart waiting list has shown no consistent trend, the number of transplants to pediatric recipients has increased marginally, from 277 in 1997 to 314 in 2006 (Figure 13). The number of pediatric deaths on the waiting list has decreased to only 59 deaths in 2006, bringing the death rate to 89 per 1000 patient-years, compared to the 2005 death rate of 122 per 1000 patient-years. One-year patient survival for pediatric heart recipients increased with increasing age from $81 \%$ for $<1$-year-olds to $91 \%$ for 11 - to 17 -year-olds (Figure 14). Among the pediatric heart transplant recipients, the 6 - to 10 -year-olds had the best 5 -year patient survival rates at $81 \%$. The prevalence of pediatric heart recipients living with a functioning graft followed the overall trend of the prevalence of all heart recipients, increasing steadily from 1369 at the end of 1997 to 2246 at the end of 2005

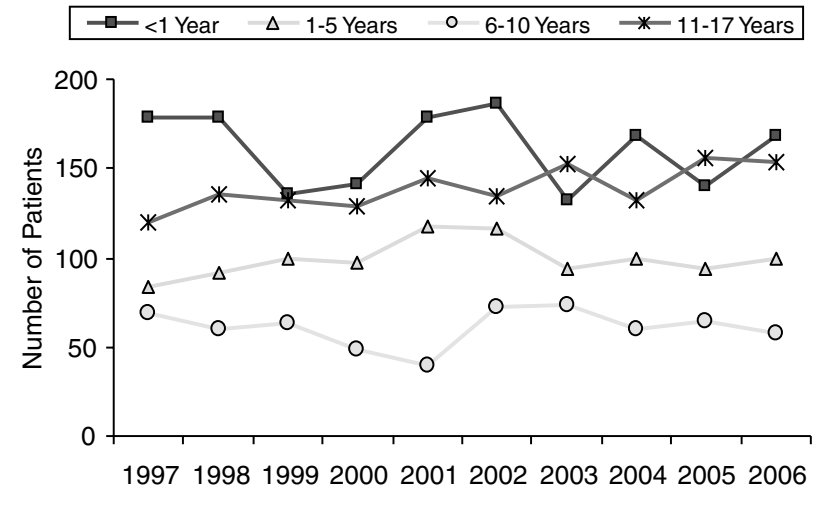

Source: 2007 OPTN/SRTR Annual Report, Table 15.3.

Figure 12: New pediatric registrants on the heart waiting list, by age, 1997-2006. 


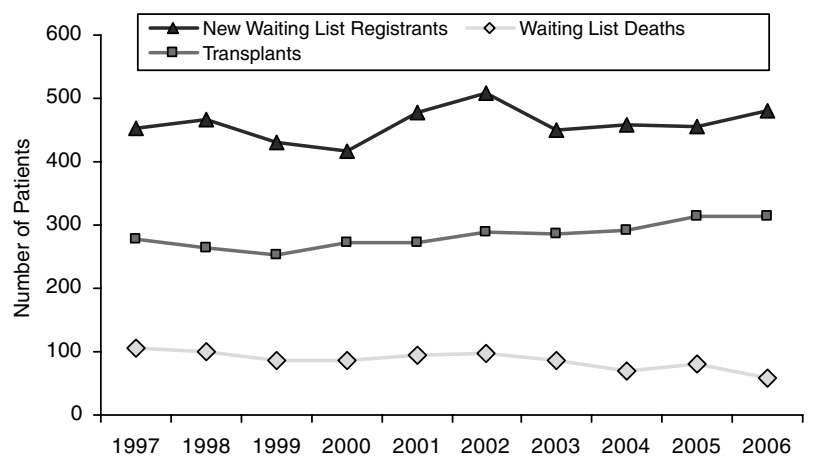

Source: 2007 OPTN/SRTR Annual Report, Tables 11.3, 11.4, and 15.3.

Figure 13: New pediatric heart waiting list registrants, deaths on the waiting list and transplants, 1997-2006.

Until recently, deceased donor hearts were allocated locally before being offered out regionally, irrespective of medical urgency. In 2006, the OPTN Board of Directors approved a new policy where deceased donor hearts would be offered to Status $1 \mathrm{~A}$ and $1 \mathrm{~B}$ candidates, first locally and then within Zone A (centers within 500 miles) prior to being offered locally to Status 2 candidates. The new policy also incorporated an element of pediatric preference for pediatric donors. The policy was implemented on 12 July 2006. While a complete evaluation of the impact of this policy change will require more follow-up, an initial analysis of 741 pediatric heart candidates spanning the pre- and postpolicy implementation periods suggests that the allocation changes have been beneficial, with a reduction in waiting list mortality following implementation of the policy $(\mathrm{HR}=$ $0.73, \mathrm{p}<0.09)$ (SRTR Analysis, October 2007).

The progress in pediatric cardiac surgery over the decade has helped shape the candidate population. Indications for pediatric heart transplant have changed as surgery for complex congenital heart lesions has evolved (9). These and other advances in the field have led the community to

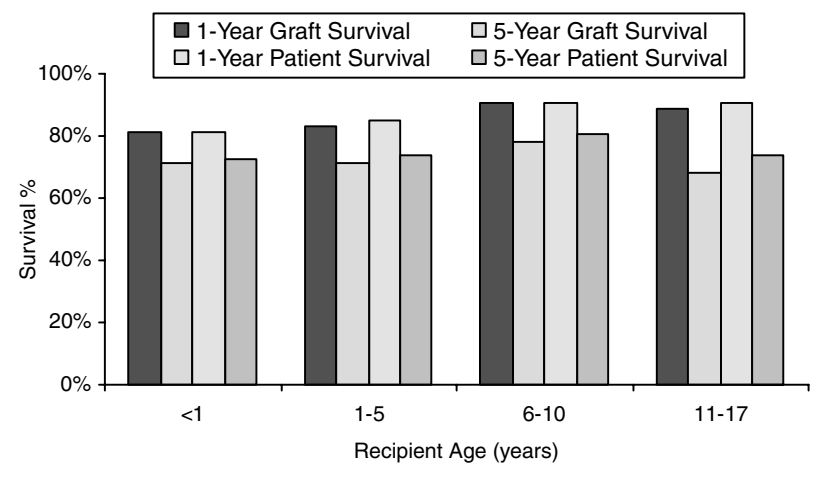

Source: 2007 OPTN/SRTR Annual Report, Tables 11.10 and 11.14 .

Figure 14: Unadjusted 1- and 5-year graft and patient survival of heart transplants, by recipient age. gather expert opinion to critically reassess the indications for heart transplantation (10).

Waiting list mortality remains high, particularly in the smallest children. The lack of suitable ventricular assist devices for use in infants as a bridge to transplantation has been a contributor to waiting list mortality in small children. Recent progress in the area has raised the possibility that such devices may become more readily available $(9,11)$.

In an effort to decrease waiting list mortality, the OPTN Pediatric committee has focused on broader sharing of pediatric donor hearts. Because donor size is critical in these smaller candidates, an appropriately sized donor may be separated by great distance from the recipient. Every effort should be made to maximize recovery of the hearts from these donors. One approach is broader sharing. Additionally, aggressive efforts at donor resuscitation and careful reevaluation over time may yield more suitable donors than previously realized (12).

An additional effort to improve waiting list survival in the smallest children is based on the observation that children under 1 year of age could be successfully transplanted with an $A B O$ incompatible heart (13). After a careful review of the available data (8), the OPTN Board voted in 2006 to allow $A B O$ incompatible heart transplants in children up to age 2 , with careful pre-transplant screening and posttransplant follow-up.

The progress in survival following heart transplantation has allowed some shifting of focus to long-term health outcomes. One of the greatest concerns is the progressive decline in renal function seen late after heart transplantation with the current calcineurin inhibitor-based immunosuppressive regimens. Using OPTN/SRTR data, Lee et al. studied pediatric heart recipients in the United States from 1990 to 1999 who survived at least 1 year after transplant and found a 10 -year actuarial risk of $4 \%$ for developing ESRD (defined as chronic dialysis and/or kidney transplant) and a 10 -year actuarial risk of $12 \%$ for developing chronic renal insufficiency (CRI, defined as creatinine $>2.5 \mathrm{mg} / \mathrm{dL}$, including those with ESRD) (14). Those who developed CRI had nine times the risk of death compared to pediatric heart recipients without $\mathrm{CRI}(\mathrm{p}<0.0001)$. Hypertension is also common, with an incidence of $47 \%$ at 1 year posttransplant (15).

\section{Lung Transplantation}

Prior to 2005, the number of pediatric lung candidates on the waiting list was relatively constant. However, this number has recently dropped precipitously from 131 in 2004 to 77 in 2005 to 42 in 2006 (Figure 15). The number of total lung candidates of all ages reduced drastically (by almost $50 \%$ ) in the past 2 years, as well, from 2163 in 2004 to 1032 in 2006. 


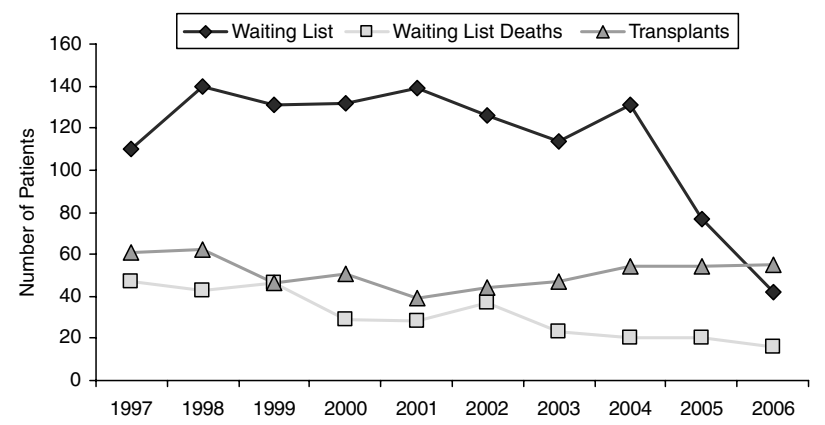

Source: 2007 OPTN/SRTR Annual Report, Tables 12.1a, 12.3 - 12.4b.

Figure 15: Pediatric patients listed for lung transplant, deaths on the waiting list and transplanted, 1997-2006.

A significant change in lung allocation policy was implemented in May 2005. Prior to that time the allocation system was based primarily on waiting time. The new allocation system is centered on the principle of transplant benefit and medical urgency. The lung allocation score (LAS) incorporates a prediction of the difference between the measures of waiting list survival and posttransplant survival for each candidate, seeking to maximize the 1-year survival benefit of lung transplantation (16). Additionally, it seeks to minimize deaths on the waiting list by balancing the benefit calculation and the degree of medical urgency, as reflected by waiting list mortality. The new allocation policies are used to distribute lungs to candidates aged 12 years and older. The decision to include adolescents with adults was based on analyses suggesting the mortality risk was very similar. In contrast, children under age 12 have a heterogeneous group of diagnoses, which makes modeling more difficult. Allocation to these younger children continues to be based on waiting time. For both the younger children and the adolescent candidates, there is also preferential allocation of pediatric donor lungs.

It is likely that the sharp reduction in the lung candidates over the last 2 years is a response to this new allocation policy where waiting time was de-emphasized. The number of pediatric deaths on the waiting list has gradually declined over the decade, as well, while the number of pediatric lung transplants per year had been relatively constant. Potential factors that may contribute to the decline in waiting list deaths include improvements in the ability to identify candidates at the highest risk of mortality and allocate lungs accordingly, refinements in management of end-stage lung disease and changes in center practice such as not listing higher risk candidates or removing candidates at the highest risk of mortality from the waiting list. The relative distribution of waiting list deaths across the pediatric age groups has stayed constant.

While the total number of lung transplants has grown $54 \%$ over the decade, from 910 in 1997 to 1401 in 2006, the

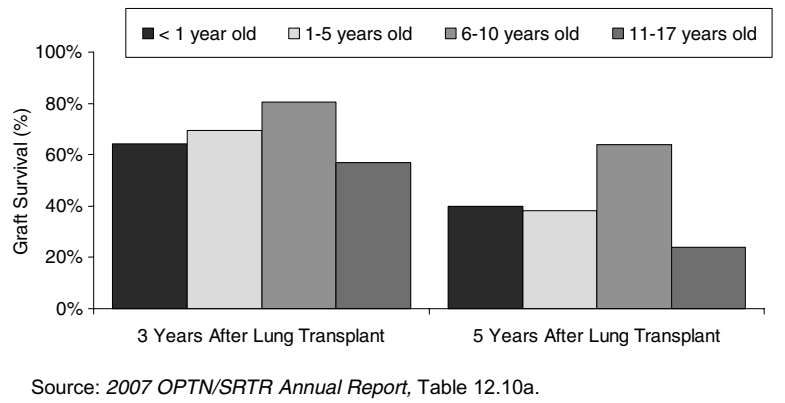

Figure 16: Unadjusted graft survival at 3 and 5 years for deceased donor lung transplant recipients, by age.

number of pediatric recipients has remained relatively stable and, consequently, the proportion of lung transplants to pediatric candidates has decreased: $3.9 \%$ of the recipients in 2006 compared to $5.8 \%$ in 1997. In 2006, 54 pediatric candidates received lung transplants and 16 died while waiting. The death rate on the lung waiting list among pediatric candidates has dropped to about 70 deaths per 1000 patient-years at risk in 2006, compared to an average of 140 deaths per 1000 patient-years in 1997-2001.

The time until $25 \%$ of the new registrants have received a lung transplant has varied widely among pediatric candidates over the past decade, from 9 to 156 days for registrants < 1-year-old, 39 to 279 days for 1 - to 5 -yearolds, 93 to 883 days for 6 - to 10 -year-olds and 40 to 783 days for 11- to 17-year-olds. At 3 years posttransplant, 6- to 10-year-old recipients of deceased donor lung transplants had the best graft survival among the pediatric age groups, at $81 \%$, followed by $<1$-year-olds at $64 \%$, then 1 - to 5 -year-olds at $70 \%$ and 11 - to 17 -year-olds at $57 \%$ (Figure 16). The deceased donor graft survival rate at 5 years was poor, especially among the 11- to 17-year-olds at $24 \%$.

At the end of 2005, 237 (4\%) of the 5684 people alive with functioning lung transplants had received their transplants as children.

Waiting list mortality has typically disproportionately affected pediatric candidates, though the finding has decreased in magnitude recently (Figure 17). Many efforts continue to be directed toward the problem of waiting list mortality. There are potential donor lungs that go unutilized. Continued focus on optimizing the management of all donors with the goal of lung donation can have a large impact on the potential number of donor lungs (17). Broader geographic sharing is also likely to better match up the appropriate donor with the appropriately sized candidate. Finally, better prioritization with respect to medical urgency is also likely to have an impact. As mentioned previously, the current LAS system does not apply to children less than 


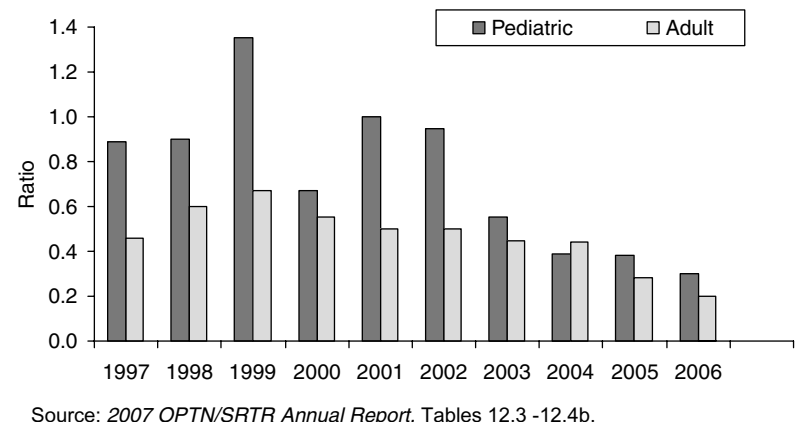

Figure 17: Ratio of lung waiting list deaths to transplants, pediatric and adult, 1997-2006.

12 years of age. Efforts to develop a LAS system in this population are hindered by the small numbers of candidates but incremental progress is possible. As one attempt to evaluate factors related to waiting list mortality, the SRTR evaluated 175 candidates less than 12 years of age who were listed between 1 January 2001 and 1 January 2003. Factors associated with an increased risk of waiting list death in a univariate analysis included being hospitalized $(\mathrm{HR}=2.69, \mathrm{p}=$ 0.0045), being in the $\operatorname{ICU}(H R=4.66, p<0.0001)$, and being on a ventilator $(H R=4.15, p=0.0002)$. Mean PA pressure did not reach statistical significance $(H R=1.02, p=0.1044)$ but it was not available in many children (SRTR Analysis, June 2007). It seems some type of urgency criteria can be developed to replace waiting time, which may get the most urgent candidates transplanted.

Pediatric lung transplantation has made significant progress but challenges remain (18). Outside the immediate postoperative period, infection accounts for half the deaths observed. At 1-year posttransplant, bronciolitis obliterans is the leading cause of death (19).

\section{Pediatric Transplantation: Common Themes}

While there are substantial differences across specific organ systems, there are also common themes across pediatric transplantation worthy of reflection. An overarching theme of the OPTN Pediatric Committee has been the charge to eliminate waiting list death in children. In liver, intestine and heart transplantation, mortality rates in the youngest candidates remain unacceptably high. Many efforts are needed to eliminate waiting list death. Encouraging donation in the pediatric population is important. We must develop protocols to ensure all potential transplantable organs are utilized.

Broader geographic sharing accomplishes many goals, including helping reduce waiting list mortality. Broader sharing of the young pediatric donor may provide a better match between the need for these organs and the availability of such donor organs, especially when combined with allocation priorities not based on waiting time.

Another theme is the profound effect changes in allocation can exert. In kidney transplantation, relative prioritization for donors less than 35 years of age has led to a substantial decrease in waiting times for pediatric candidates. One potential unintended consequence of this increased priority is a decrease in the numbers of living donor transplants performed. Additionally, the removal of waiting time goals for pediatric recipients may be adversely affecting the highly sensitized pediatric recipient who now may have decreased access to the larger pool of donors who are above age 35. Efforts are underway to better evaluate both of these issues. In liver and lung transplantation, a switch from allocation based on waiting time to one based on urgency has profoundly affected listing practices.

The impact of growth and development on outcomes remains poorly understood. Graft loss in adolescent recipients is a problem. Nonadherence is the most commonly cited factor yet there are other mechanisms, including physiologic growth and development, which may be involved (20). As pediatric transplant recipients, they have both a lifetime of hope and a lifetime of care. The financial pressures involved, and the impact of finances on outcomes, should not continue to go unanswered $(21,22)$

The final issue concerns data collection. In 2006, the OPTN embarked on an effort to decrease the data reporting burden, focusing specifically on areas of redundancy and on long-term (greater than 5 years posttransplant) followup. It has been estimated that these changes have led to an approximate $40 \%$ reduction in the data reporting burden. During this process, the pediatric community expressed concern that much of the benefit of transplantation in pediatric candidates relates to long-term outcomes and these data are vital. Additionally, much of the data of interest in children is different compared to adults. Because of this, it has been recognized that there are data elements that need revision to fully serve the pediatric population. The OPTN Board has been receptive to these concerns.

While the reporting requirements for pediatric patients remain largely intact, there is a clear mandate that alternative sources of information be explored and supported, and wherever possible duplication of existing efforts be avoided. It is noteworthy that there are pediatric-specific transplant registries (e.g. NAPRTCS or SPLIT) that might serve as parallel sources of data. In the past, these registries have played a fundamental role in providing data for analysis used in the development of pediatric allocation policy. Future funding for these registries is uncertain and these data collection efforts may not be able to continue without partnership with the OPTN. How to best link the 
OPTN data collection needs and the interests of these pediatric registries, and how this effort would be supported, is worthy of discussion.

\section{Acknowledgments}

The SRTR is funded by contract number 234-2005-37009C from the Health Resources and Services Administration (HRSA), US Department of Health and Human Services. The views expressed herein are those of the authors and not necessarily those of the US Government. This is a US Governmentsponsored work. There are no restrictions on its use.

This study was approved by HRSA's SRTR project officer. HRSA has determined that this study satisfies the criteria for the IRB exemption described in the 'Public Benefit and Service Program' provisions of 45 CFR 46.101 (b)(5) and HRSA Circular 03

This article was produced as part of the 2007 OPTN/SRTR Annual Report. The Annual Report gathers information on many aspects of solid organ transplantation in one publication. More information can be found at www.ustransplant.org.

Note on sources: The articles in this report are based on the reference tables in the 2007 OPTN/SRTR Annual Report, which are not included in this publication. Many relevant data appear in the figures and tables included here; other tables from the Annual Report that serve as the basis for this article include the following: Tables 1.4, 1.10, 2.1, 2.2, 2.3 $2.4,2.5,2.6,2.7,5.1 a, 5.4 a, 5.4 c, 5.10 a, 5.10 c, 5.14 a, 5.14 c, 5.16,9.1 a$, $9.1 b, 9.3,9.4 a, 9.4 b, 9.7 b, 9.10 a, 9.10 b, 9.14 a, 9.14 b, 9.16,10.1 a, 10.3$ $10.4,10.10,10.14,10.16,11.1 \mathrm{a}, 11.3,11.4,11.14,11.16,12.1 \mathrm{a}, 12.2,12.3$ $12.4 \mathrm{a}, 12.4 \mathrm{~b}, 12.10 \mathrm{a}, 12.16,15.3$. All of these tables may be found online at: www.ustransplant.org.

\section{References}

1. Durall AL, Laussen PC, Randolph AG. Potential for donation after cardiac death in a children's hospital. Pediatrics 2007; 119: e219e224.

2. McDonald RA, Guidinger MK, McCullough KP et al. Simulation modeling of preferential allocation of kidneys from donors less than age 35 years to pediatric candidates. Am J Transplant 2005; 5(11 Suppl): 281 (abstract).

3. Organ procurement and transplantation network: Policies. Available from: http://www.optn.org/policiesAndBylaws/policies.asp, accessed September 12, 2006.

4. Horslen S, Barr ML, Christensen LL, Ettenger R, Magee JC. Pediatric transplantation in the United States, 1996-2005. Am J Transplant 2007; 7(5 Pt 2): 1339-1358.

5. Gritsch HA, McDonald R, Leichtman A et al. Should pediatric patients wait for an HLA-DR matched kidney? Am J Transplant 2005; 5(11 Suppl): 401 (abstract).
6. Merion RM, Rush SH, Dykstra DM, Goodrich N, Freeman RB Jr., Wolfe RA. Predicted lifetimes for adult and pediatric split liver versus adult whole liver transplant recipients. Am J Transplant 2004; 4: 1792-1797.

7. Roberts JP, Hulbert-Shearon TE, Merion RM, Wolfe RA, Port FK. Influence of graft type on outcomes after pediatric liver transplantation. Am J Transplant 2004; 4: 373-377.

8. Sweet SC, Wong HH, Webber SA et al. Pediatric transplantation in the United States, 1995-2004. Am J Transplant 2006; 6(5 Pt 2): 1132-1152.

9. Alkhaldi A, Chin C, Bernstein D. Pediatric cardiac transplantation. Sem Pediatr Surg 2006; 15: 188-198.

10. Canter CE, Shaddy RE, Bernstein D et al. Indications for heart transplantation in pediatric heart disease: A scientific statement from the American Heart Association Council on cardiovascular disease in the young; the councils on clinical cardiology, cardiovascular nursing, and cardiovascular surgery and anesthesia; and the quality of care and outcomes research interdisciplinary working group. Circulation 2007; 115: 658-676.

11. Potapov EV, Stiller B, Hetzer R. Ventricular assist devices in children: Current achievements and future perspectives. Pediatr Transplant 2007; 11: 241-255.

12. Audibert G, Charpentier C, Seguin-Devaux C et al. Improvement of donor myocardial function after treatment of autonomic storm during brain death. Transplantation 2006; 82: 1031-1036.

13. West LJ, Pollock-Barziv SM, Dipchand Al et al. ABO-incompatible heart transplantation in infants. N Engl J Med 2001; 344: 793-800.

14. Lee CK, Christensen LL, Magee JC, Ojo AO, Harmon WE, Bridges ND. Pre-transplant risk factors for chronic renal dysfunction after pediatric heart transplantation: a 10-year national cohort study. $J$ Heart Lung Transplant 2007; 26: 458-465.

15. Boucek MM, Aurora P, Edwards LB et al. Registry of the international society for heart and lung transplantation: Tenth official pediatric heart transplantation report-2007. J Heart Lung Transplant 2007; 26: 796-807.

16. Egan TM, Murray S, Bustami RT et al. Development of the new lung allocation system in the United States. Am J Transplant 2006; 6(5 Pt 2): 1212-1227.

17. Studer SM, Orens JB. cadaveric donor selection and management Sem Resp Crit Care Med 2006; 27: 492-500.

18. Wells A, Faro A. Special considerations in pediatric lung transplantation. Sem Resp Crit Care Med 2006; 27: 552-560.

19. Waltz DA, Boucek MM, Edwards LB et al. Registry of the international society for heart and lung transplantation: Ninth official pediatric lung and heart-lung transplantation report. J Heart Lung Transplant 2006; 25: 904-911.

20. Hsu DT. Biological and psychological differences in the child and adolescent transplant recipient. Pediatr Transplant 2005; 9: 416421.

21. Willoughby LM, Fukami S, Bunnapradist $S$ et al. Health insurance considerations for adolescent transplant recipients as they transition to adulthood. Pediatr Transplant 2007; 11: 127-131.

22. Benfield M. Insurance, non-adherence-a call to action. Pediatr Transplant 2007; 11: 236-238. 